\title{
The BigLEN-GPR I7I Peptide Receptor System Within the Basolateral Amygdala Regulates Anxiety-Like Behavior and Contextual Fear Conditioning
}

\author{
Erin N Bobeck ${ }^{*, I}$, Ivone Gomes', Darlene Pena ${ }^{2}$, Kirstie A Cummings ${ }^{3}$, Roger L Clem $^{3}$, Mihaly Mezei' and \\ Lakshmi A Devi*,i \\ 'Department of Pharmacological Sciences, Icahn School of Medicine at Mount Sinai, New York, NY, USA; '2Departamento de Bioquímica, Instituto \\ de Química, Universidade de São Paulo, São Paulo, Brazil; ${ }^{3}$ Fishberg Department of Neuroscience, Icahn School of Medicine at Mount Sinai, New \\ York, NY, USA
}

\begin{abstract}
Studies show that neuropeptide-receptor systems in the basolateral amygdala (BLA) play an important role in the pathology of anxiety and other mood disorders. Since GPR I7I, a recently deorphanized receptor for the abundant neuropeptide BigLEN, is expressed in the BLA, we investigated its role in fear and anxiety-like behaviors. To carry out these studies we identified small molecule ligands using a homology model of GPR I 7 I to virtually screen a library of compounds. One of the hits, MS002 I570_I, was identified as a GPR I7I antagonist based on its ability to block (i) BigLEN-mediated activation of GPRI7I in heterologous cells, (ii) BigLEN-mediated hyperpolarization of BLA pyramidal neurons, and (iii) feeding induced by DREADD-mediated activation of BigLEN containing AgRP neurons in the arcuate nucleus. The role of GPRI7I in anxiety-like behavior or fear conditioning was evaluated following systemic or intra-BLA administration of MS0021570_I, as well as following lentiviral-mediated knockdown of GPRI7I in the BLA. We find that systemic administration of MS0021570_I attenuates anxiety-like behavior while intra-BLA administration or knockdown of GPRI7I in the BLA reduces anxiety-like behavior and fear conditioning. These results indicate that the BigLEN-GPRI7I system plays an important role in these behaviors and could be a novel target to develop therapeutics to treat psychiatric disorders.

Neuropsychopharmacology (2017) 42, 2527-2536; doi:I0.1038/npp.2017.79; published online 24 May 2017
\end{abstract}

\section{INTRODUCTION}

Mental health disorders such as anxiety and post-traumatic stress disorder affect $\sim 11 \%$ of the population (Silberberg et al, 2015). Despite years of research, current treatments for these disorders are limited and associated with numerous side effects. Thus, alternative therapies for these disorders are needed. Neuropeptide receptors are ideally suited for the development of therapeutics due to their involvement in different physiological behaviors including fear, anxiety, depression, and pain. We recently identified GPR171 as the receptor for BigLEN, one of the most abundant neuropeptides in the brain (Fricker, 2010), and found that the BigLENGPR171 system modulates feeding-related behaviors within the hypothalamus (Gomes et al, 2013). However, not much is known about the role of BigLEN-GPR171 system in other brain regions and other physiological processes.

*Correspondence: Dr EN Bobeck or Dr LA Devi, Department of Pharmacological Sciences, Icahn School of Medicine at Mount Sinai, One Gustave L Levy Place, Annenberg 19-84, New York, NY 10029, USA. Tel: + I 212 24|8345, Fax: + I 212 9967214,

E-mail: erin.bobeck@mssm.edu or lakshmi.devi@mssm.edu

Received I5 December 2016; revised 9 April 2017; accepted 12 April 2017; accepted article preview online 20 April 2017
The Allen Brain Atlas shows expression of BigLEN and GPR171 in the basolateral amygdala (BLA) (Lein et al, 2007). The BLA plays a critical role in anxiety-like behaviors and in memory acquisition, consolidation, and retrieval by processing affective information and attaching emotional significance to cues and events (Stamatakis et al, 2014). Neuropeptide-receptor systems are thought to modulate these behaviors in the amygdala. For example, Neuropeptide $\mathrm{Y}$ (NPY), a peptide involved in regulation of feeding in the hypothalamus, has been shown to produce anxiolytic effects and lead to resilience after stress (Sajdyk et al, 2008; Desai et al, 2013; Christiansen et al, 2014).

Since we find expression of BigLEN and GPR171 in the BLA, we hypothesized that this peptide-receptor system could play a role in regulating mood-related behaviors. To test this hypothesis, we identified a small molecule antagonist by in silico screening of a homology model. We used molecular and behavioral pharmacology and electrophysiology to characterize this compound within the BLA. In addition, we confirmed the physiological involvement of GPR171 in anxiety-like and fear behaviors using shRNAmediated reduction in GPR171 in this brain region. Our studies indicate that GPR171 represents a novel target for the development of novel therapeutics to treat psychiatric disorders. 


\section{MATERIALS AND METHODS}

\section{Subjects}

Male C57bl/6 J or AgRP-IRES-Cre mice (Jackson Laboratories) were 8-12 weeks old at the beginning of the experiment. Food and water were available ad libitum except during testing. Mice were group housed (4-5 per cage) in a humidity and temperature-controlled room with a $12 \mathrm{~h}$ light/ dark cycle (on 0700-1900 hours). Testing took place between 0800 and 1600 hours. All procedures were approved by the Institutional Care and Use Committee at Icahn School of Medicine at Mount Sinai.

\section{Surgical Procedures}

Mice were anesthetized with ketamine/xylazine $(100 \mathrm{mg} / \mathrm{kg}$, i.p.). Control or GPR171 shRNA lentivirus $(0.5 \mu \mathrm{l}$, rate of $0.1 \mu \mathrm{l} / \mathrm{min}, 10^{-9}$ (Gomes et al, 2013)) was infused into the BLA (AP: $-1.7 \mathrm{~mm}, \mathrm{ML}: \pm 3 \mathrm{~mm}$, DV: $-4.8 \mathrm{~mm}$ ) using stereotaxical techniques (Paxinos and Franklin, 2001). The injector was kept in place for an additional 2 min to prevent backflow. To selectively activate AgRP neurons a credependent adeno-associated virus excitatory DREADD ( $0.5 \mu \mathrm{l}$, rate of $0.1 \mu \mathrm{l} / \mathrm{min}, \mathrm{AAV} 8-\mathrm{hSyn}-\mathrm{DIO}-\mathrm{hM} 3 \mathrm{Dq}$-mCherry; University North Carolina vector core, $10^{-9}$ ) was bilaterally infused into the arcuate nucleus (AP: $-1.5 \mathrm{~mm}$, ML: $\pm 0.3 \mathrm{~mm}, \mathrm{DV}:-5.9 \mathrm{~mm}$ ) of AgRP-IRES-Cre mice. For microinjection studies, mice were bilaterally cannulated into the BLA (24 gauge, $6 \mathrm{~mm}$ ). Injection cannulae were inserted and extended $1 \mathrm{~mm}$ past the guide cannula. Mice were allowed to recover 2 weeks prior to beginning behavioral testing. Microinjections of vehicle (10\% DMSO, $10 \%$ Tween80 in saline) or MS0021570_1 $(20 \mathrm{nmol} / 0.5 \mu \mathrm{l})$ were administered $0.5 \mu \mathrm{l}$ over $1 \mathrm{~min}$.

\section{Behavioral Assays}

Mice were habituated to the testing room $1 \mathrm{~h}$ prior to each experiment. Behaviors were videotaped and analyzed using Noldus EthoVision XT or by an experimenter blinded to the treatment groups. Mice were injected with MS0021570_1 ( $5 \mathrm{mg} / \mathrm{kg}$, i.p.) $15 \mathrm{~min}$ prior to elevated plus maze, open field, forced swim, and contextual fear conditioning. For microinjection studies, MS0021570_1 was injected 10 min prior to testing and immediately following the contextual fear conditioning. A counterbalanced within-subjects design was used when appropriate (ie, battery of anxiety tests).

Food intake. Singly housed AgRP-IRES-Cre mice with creinducible DREADD in the arcuate nucleus received MS0021570_1 (3.5 mg/kg, i.p.) or vehicle (6\% DMSO in saline) $15 \mathrm{~min}$ prior to $\mathrm{CNO}(1 \mathrm{mg} / \mathrm{kg}$, i.p.) or saline injection. Food intake was measured at 1,2, 4, and $8 \mathrm{~h}$ post injection.

Forced swim test. Mice were placed into a 41 glass beaker with 2.51 water $\left(25 \pm 1{ }^{\circ} \mathrm{C}\right)$ for $6 \mathrm{~min}$. Immobility was scored using a $5 \mathrm{~s}$ sampling method as previously published (Hodes et al, 2015) and the percentage of time spent immobile was reported.
Elevated plus maze. The apparatus consisted of two open and two closed arms $(12 \times 50 \mathrm{~cm}$ each $)$ on a pedestal $60 \mathrm{~cm}$ above the floor. Mice explored the maze for $5 \mathrm{~min}$ under red light and amount of time spent in each arm was tracked. Data are presented as open arm time/(open arm+closed arm time).

Contextual fear conditioning. Mice were placed into a chamber with steel rod flooring and white noise for $2 \mathrm{~min}$, then received a $2 \mathrm{~s}$ shock $(0.6 \mathrm{~mA})$ followed by an additional 2 min with white noise. The following day mice were placed back into the same chamber and time spent freezing was measured for $5 \mathrm{~min}$ using a $5 \mathrm{~s}$ sampling method. A subset of mice was tested at 1 and 2 weeks post shock.

\section{Immunohistochemistry}

Immunohistochemistry was conducted as described previously (Wardman et al, 2016). More details are in the Supplementary Material and Methods. Briefly, staining was performed on free-floating coronal slices $(40 \mu \mathrm{m})$ containing BLA from $4 \%$ paraformaldehyde-perfused mice. Primary antibodies were used to detect GPR171 $(1: 250$, Genetex rabbit) or GFP (1:500, mouse, Cell Signaling). Microscopy was performed at the Microscopy CORE at the Icahn School of Medicine at Mount Sinai using either Leica DM6000 or Leica SP5-DM confocal. Cannula placements were determined using injection of trypan blue and detection with a light microscope.

\section{Current-Clamp Recordings}

More details on slice preparation and electrophysiology in Supplementary Material and Methods. Briefly, slices containing BLA were prepared in high-sucrose cutting solution (in $\mathrm{mM}$ : 210.3 sucrose, $26.2 \mathrm{NaHCO}_{3}, 11$ glucose, $4 \mathrm{MgCl}_{2}$, $2.5 \mathrm{KCl}, 1 \mathrm{NaH}_{2} \mathrm{PO}_{4}, 0.5$ ascorbate, and $0.5 \mathrm{CaCl}_{2} ;-2$ to $-4{ }^{\circ} \mathrm{C}$ ). Slices were maintained in standard artificial cerebrospinal fluid (ACSF, in mM: $119 \mathrm{NaCl}, 26.2 \mathrm{NaHCO}_{3}$, 11 glucose, $2.5 \mathrm{KCl}, 2 \mathrm{CaCl}_{2}, 2 \mathrm{MgCl}_{2}$, and $1 \mathrm{NaH}_{2} \mathrm{PO}_{4}$ ) saturated with $5 \% \mathrm{CO}_{2} / 95 \% \mathrm{O}_{2}$.

Electrodes were 3-5 M 2 when filled with intracellular solution containing (in $\mathrm{mM}$ ): $127.5 \mathrm{~K}$-methanesulfonate, 10 HEPES, $5 \mathrm{KCl}, 5 \mathrm{Na}$-phosphocreatine, $2 \mathrm{MgCl}_{2}, 2 \mathrm{Mg}$-ATP, 0.6 EGTA, and $0.3 \mathrm{NaGTP}$ (pH 7.25; 285-300 mOsm). Pyramidal neurons in the BLA were visually identified based on morphology. Cells were maintained at $-70 \mathrm{mV}$ with current injection (MultiClamp 700B, Molecular Devices, Sunnyvale, CA) and membrane potential was continuously recorded using a gap-free protocol in Clampex (Molecular Devices). For BigLEN experiments, a stable baseline was established ( $\geqslant 3 \mathrm{~min}$ ) in ACSF, followed by a switch to ACSF $+100 \mathrm{nM}$ BigLEN. Following equilibration to steady state, bath solution was changed to ACSF $+100 \mathrm{nM}$ BigLEN $+1 \mu \mathrm{M}$ antagonist (MS0021570_1); these solutions had a final concentration of $1 \times$ protease inhibitor cocktail and $0.0001 \%$ DMSO. For small molecule agonist experiments, $1 \mu \mathrm{M}$ agonist (MS0015203) was used in place of BigLEN; solutions for these experiments had a final concentration of $1 \times$ protease inhibitor cocktail and $0.0002 \%$ DMSO. Solutions were delivered using a homemade gravity perfusion system. Data were sampled at $10 \mathrm{kHz}$ and low pass filtered at 
$3 \mathrm{kHz}$. Traces were analyzed in Clampfit (Molecular Devices).

\section{Biochemical Assays}

Detailed protocols are in Supplementary Material and Methods.

Materials. Mouse GPR171 cDNA from Open Biosystems was subcloned into pCMV-myc-N-terminal Epitope Tagging Mammalian Expression Vector (Stratagene) according to the manufacturer's protocol. Chinese hamster ovary $(\mathrm{CHO})$ cells were from ATCC. F-12 media was from Gibco. Lipofectamine and Fluo4NW calcium dye were from Invitrogen. FBS was from Biowest. Pierce iodination reagent was from Thermo Scientific. Na ${ }^{125}$ I and $\left[{ }^{35} \mathrm{~S}\right] \mathrm{GTP} \gamma \mathrm{S}$ were from PerkinElmer. Protease inhibitor cocktail, ATP were from Sigma-Aldrich. DYKDDDDK-tagged mGPR171 or mouse melatonin $1 \mathrm{~A}$ (mMT1A) receptor cDNAs were from GenScript. MS0016911 was from Chembridge and MS0022269 from InterBioscreen. MS0015917 (10 mM aliquot) was from the screening library at Icahn School of Medicine at Mount Sinai.

Cell culture and transfections. $\mathrm{CHO}$ cells grown in F-12 medium containing $10 \%$ fetal bovine serum and $1 \times$ penicillin-streptomycin were transfected with $m y c$-tagged GPR171 along with a chimeric $\mathrm{G}_{\alpha 15 / \mathrm{i} 3}$ protein using lipofectamine as per the manufacturer's protocol for experiments measuring intracellular $\mathrm{Ca}^{+2}$ levels. $\mathrm{CHO}$ cells were transfected with DYKDDDDK-tagged mGPR171 or mMT1A receptor constructs using lipofectamine and stable cell lines generated as described in the manufacturer's protocol.

Homology modeling and in silico screening at GPR171. A homology model of mouse GPR171 based on the crystal structure of the phylogenetically related P2Y12 receptor (Gloriam et al, 2007; Kakarala and Jamil, 2014) corresponding to PDB code 4PXZ (Piccoli et al, 2014) was generated as described previously (Wardman et al, 2016). This homology model was used for in silico screening of small molecule ligands for GPR171 as described previously (Wardman et al, 2016).

Measurement of intracellular $\mathrm{Ca}^{+2}$ levels. These assays were carried out as described previously (Gomes et al, 2009; Gupta et al, 2010; Gomes et al, 2013) using CHO cells (40 000 cells per well) expressing $m y c$-tagged mGPR171 along with a chimeric $\mathrm{G}_{\alpha 15 / \mathrm{i} 3}$ protein treated with $1 \mu \mathrm{M}$ BigLEN in the absence or presence of $10 \mu \mathrm{M}$ of the 13 compounds from the screening library. Increases in intracellular $\mathrm{Ca}^{+2}$ levels were measured for $\sim 210 \mathrm{~s}$ at excitation $494 \mathrm{~nm}$ and emission $516 \mathrm{~nm}$.

Membrane preparation. Membranes from rat hypothalamus were prepared as described previously (Gomes et al, 2003, 2013).

Binding assays. Tyr-BigLEN $(200 \mu \mathrm{g})$ was iodinated using $\left[{ }^{125} \mathrm{I}\right]$ and Pierce iodination reagent as described in the manufacturer's protocol (Thermo Scientific). The specific activity of the iodinated peptide was $65.3 \mathrm{Ci} / \mathrm{mmol}$ at the time of iodination (the radiolabelled peptide was used within 60 days of iodination). Binding assays were carried out as described previously (Gomes et al, 2003, 2013; Rozenfeld et al, 2011) using $3 \mathrm{nM}$ of $\left[{ }^{125} \mathrm{I}\right] \mathrm{Tyr}$-BigLEN and different concentrations $(0-10 \mu \mathrm{M})$ of BigLEN (LENPSPQAPARRLLPP), MS0021570_1, MS0015917, MS0016911, or MS0022269.

$\left[{ }^{35}\right.$ S $]$ GTP $\gamma$ S binding. $\left[{ }^{35} \mathrm{~S}\right] \mathrm{GTP} \gamma \mathrm{S}$ binding assays were carried out as described previously (Gupta et al, 2010; Rozenfeld et al, 2011; Gomes et al, 2013) using membranes from rat hypothalamus $(10 \mu \mathrm{g})$ incubated for $1 \mathrm{~h}$ at $30^{\circ} \mathrm{C}$ with $1 \mu \mathrm{M}$ BigLEN or DAMGO in the absence or presence of MS0021570_1 $(0-10 \mu \mathrm{M})$, or with MS0015203, $(0-10 \mu \mathrm{M}$ final concentration) in the absence or presence of $10 \mu \mathrm{M}$ MS0021570_1.

Determination of cAMP levels. $\mathrm{CHO}$ cells expressing either DYKDDDDK-tagged mGPR171, mP2Y12, or mMT1A receptor (10000 cells per well), or homogenates from rat amygdala were pretreated for $30 \mathrm{~min}$ with $20 \mu \mathrm{M}$ forskolin followed by treatment with either BigLEN $(1 \mu \mathrm{M})$, MS0015203 $(1 \mu \mathrm{M})$, MeSADP $(100 \mathrm{nM})$, or melatonin $(100 \mathrm{nM})$ in the absence or presence of MS0021570_1 $(10 \mu \mathrm{M})$ for $30 \mathrm{~min}$. cAMP levels were quantified using the HitHunter cAMP detection kit from DiscoveRx according to the manufacturer's protocol. Due to the size of tissue, homogenates of rat amygdala were used.

$q R T-P C R$. Brains were removed and $2 \mathrm{~mm}$ slices were taken $\sim 1 \mathrm{~mm}$ caudal from Bregma. Punches of BLA were taken using $1 \mathrm{ml}$ pipette tip. RNA extraction and cDNA preparation was performed as described previously (Wardman et al, 2016) from BLA of mice expressing control or lentiviral shRNA targeting GPR171. Quantitative RT-PCR was performed at the Icahn School of Medicine at Mount Sinai qPCR core with ABI PRISM 7900HT detection system. Analysis was performed using the $\Delta \Delta \mathrm{C}_{\mathrm{T}}$ method.

Data and statistical analyses. Results are expressed as the mean \pm SE. Data comparing more than one group were analyzed using one-way ANOVAs or two-way ANOVAs (repeated measures when appropriate) followed by Fisher's LSD or Bonferroni's post hoc comparisons. Data comparing two groups were analyzed using two-tailed unpaired Student's $t$-tests. Significance was set at $p<0.05$. Statistical analyses of data were generated by using Prism software (version 6.0; GraphPad Software).

\section{RESULTS}

\section{Identification and Characterization of a Small Molecule Antagonist to GPR171}

Using a homology model of GPR171 to virtually dock an inhouse library of chemical compounds, we previously identified 13 putative small molecule ligands that when tested in a cell-based assay measuring intracellular $\mathrm{Ca}^{+2}$ release in cells expressing GPR171 and a chimeric $\mathrm{G}_{15 / \mathrm{i} 3}$ 
a

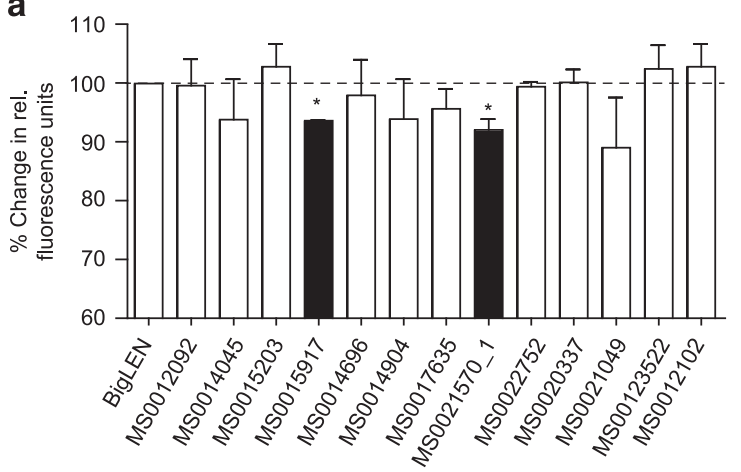

C

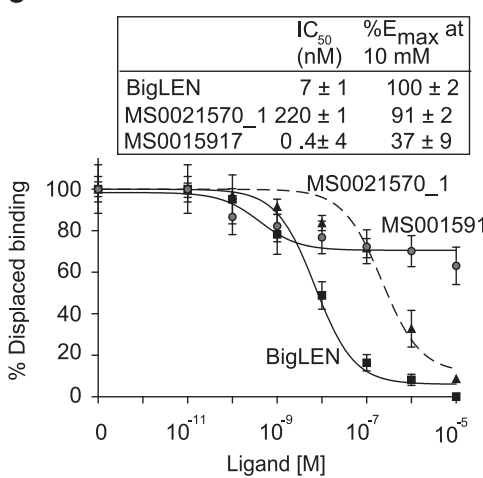

d

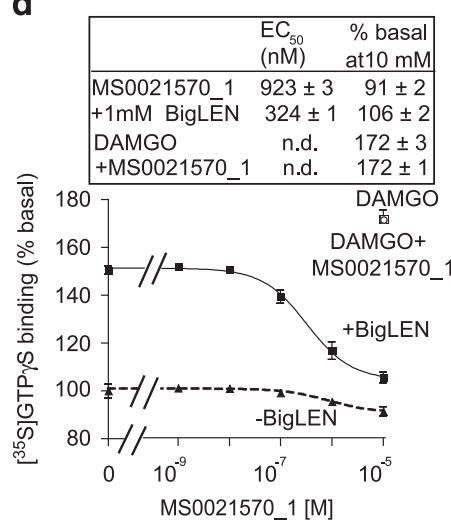

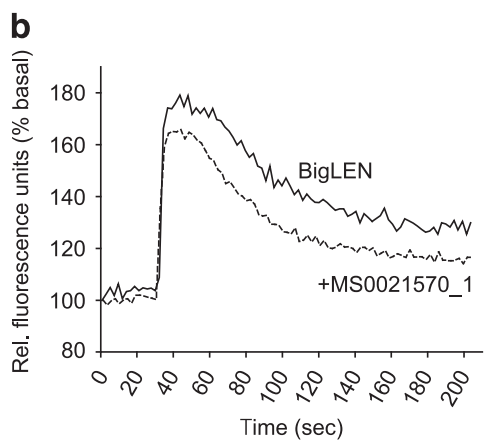

e
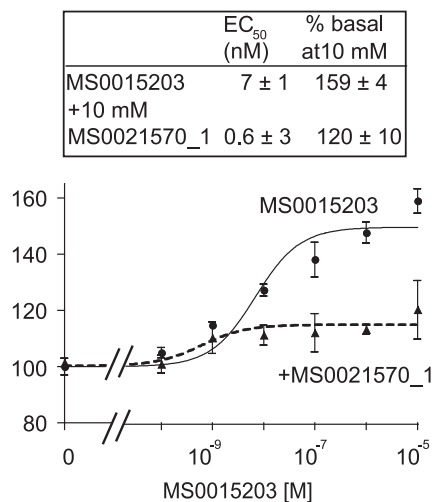

f

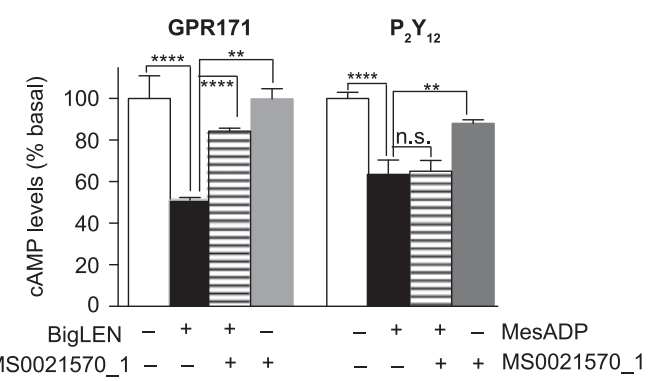

Figure I Characterization of MS0021570_I as an antagonist of GPRI7I. (a) Of the ligands predicted by in silico screening to bind to GPRI7I only MSO0 I59I7 and MS0021570_I (I0 MM) inhibit increases in $\mathrm{Ca}^{+2}$ levels induced by BigLEN (I HM) in CHO cells expressing GPRI7I and a chimeric GI5/i3 protein. * $p<0.05$, one-way ANOVA. (b) Representative plot of inhibition of BigLEN-mediated increases in intracellular $\mathrm{Ca}^{+2}$ levels by MS002I570_I is shown. (c) MS002 1570_I dose dependently displaced [ ${ }^{125}$ I]Tyr-BigLEN binding in rat hypothalamic membranes albeit with lower affinity than the native ligand, BigLEN. Binding in the absence of cold ligands was taken as 100\%. (d) MS0021570_I dose dependently antagonized I HM BigLEN-mediated increases in ${ }^{35} \mathrm{~S}$ ] GTP $\gamma$ S binding in rat hypothalamic membranes. At high concentrations, MS0021570_l exhibits a small but not significant inverse agonistic activity.

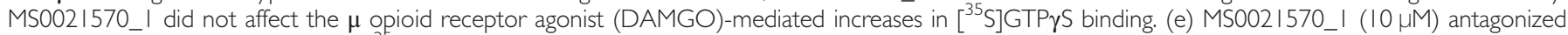

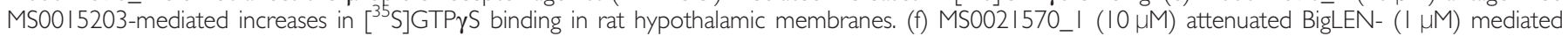
decreases in cAMP levels in CHO cells expressing GPR I7I. MS002 I570_I ( $10 \mu M)$ had no effect on MesADP- (I00 nM) mediated decreases in cAMP levels in $\mathrm{CHO}$ cells expressing mP2YI 2 receptor. $* * * 0.01$; ****** $p<0.000$ I, one-way ANOVA. Data in a-f represent means $\pm S E(n=3-6)$. ANOVA, analysis of variance; $\mathrm{CHO}$, Chinese hamster ovary; ND, not detected; NS, not significant.

protein identified MS0015203 as a GPR171 agonist (Wardman et al, 2016). To identify a receptor antagonist, we tested these compounds in the presence of BigLEN and found two of them (MS0021570_1 and MS0015917) to be antagonists as evidenced by significant $(p<0.05)$ attenuation of BigLEN-mediated increases in intracellular $\mathrm{Ca}^{+2}$ release (Figure 1a and b). Displacement binding assays show that MS0021570_1 dose dependently and completely displaced radiolabeled BigLEN binding to hypothalamic membranes albeit with a lower affinity ( $\sim 31$-fold) compared to BigLEN (Figure 1c); MS0015917 only partially ( 37\%) displaced radiolabeled BigLEN binding (Figure 1c). Also, MS0016911 and MS0022269, two other compounds belonging to the same cluster as MS0021570_1 (that could be commercially purchased) were less potent and not able to displace radiolabeled BigLEN to the same extent as MS0021570_1 (Supplementary Table S1). Thus, we focused our characterization studies on MS0021570_1.

\section{Characterization of MS0021570_1 as a Possible Selective Antagonist for GPR171}

Next, we examined the properties of MS0021570_1 in the G-protein activity assay. MS0021570_1 dose dependently 
decreased the agonist- (BigLEN, endogenous peptide or MS0015203, synthetic small molecule) mediated increases in $\left[{ }^{35} \mathrm{~S}\right] \mathrm{GTP} \gamma \mathrm{S}$ binding to rat hypothalamic membranes (Figure 1d and e). Interestingly, on its own MS0021570_1 did not exhibit agonistic activity and exhibited only a slight inverse agonistic activity at very-high concentrations (Figure 1d). Moreover, MS0021570_1 did not antagonize the activity of an unrelated G-protein coupled receptor ( $\mu$-opioid receptor; Figure 1d). MS0021570_1 also behaved as an antagonist in the inhibition of adenylyl cyclase activity since we found that MS0021570_1 significantly attenuated BigLEN-mediated decreases in intracellular cAMP levels (Figure 1f). In contrast, in cells expressing the $\mathrm{P} 2 \mathrm{Y} 12$ receptor (the receptor used for homology modeling), MS0021570_1 had no significant effect on its own or on the P2Y12 agonist (MesADP)-mediated decreases in cAMP levels (Figure 1f). These results indicate that MS0021570_1 exhibits antagonistic activity at GPR171.

To assess the level of selectivity of MS0021570_1 for GPR171, the ligand was screened against a panel of 80 different membrane targets (including $~ 70$ GPCRs). The ability of MS0021570_1 to displace radioligand binding to each of the targets was tested at $10 \mu \mathrm{M}$ concentration; at this concentration MS0021570_1 displaced $<30 \%$ binding at these targets (Supplementary Figure S1A), with the exception of melatonin 1A receptor (MT1AR). We directly examined the effect of MS0021570_1 on MT1AR and found that MS0021570_1 exhibited a 1000-fold lower potency in the cAMP assays and did not further affect MT1AR agonistmediated decreases in cAMP (Supplementary Figure S1B and C). These results suggest that MS0021570_1 is a GPR171 antagonist at low (submicromolar) doses and at very-high doses it has the potential to function as an MT1AR agonist.

\section{In vivo Characterization of GPR171 Antagonist}

To study MS0021570_1's antagonistic activity in vivo, we examined if it has the ability to inhibit BigLEN-/GPR171mediated effects. For this, we used an excitatory DREADD to specifically examine the effect of MS0021570_1 to block the activation of AgRP neurons; BigLEN has been shown to be coexpressed in neurons that contain AgRP/NPY in the arcuate nucleus and activation of these neurons is likely to lead to the release of BigLEN (Wardman et al, 2011). MS0021570_1 did not alter feeding over $8 \mathrm{~h}$ in mice on normal chow diet (Supplementary Figure S2A; $t_{(19)}=1.54$; $p=0.14)$. We expressed the excitatory DREADD in the AgRP neurons (Supplementary Figure S2B) and examined the effect of the antagonist on CNO-mediated increase in food intake. As shown previously, activating AgRP neurons with $\mathrm{CNO}$ led to a significant increase in feeding compared to saline controls (Krashes et al, 2011); Supplementary Figure S2C; $\left.\mathrm{F}_{(1,123)}=26.82 ; p<0.001\right)$ and this correlated with the number of neurons expressing the DREADD (Supplementary Figure S2D; $r=0.56, p<0.05$ ). Importantly, we find that pretreatment with the GPR171 antagonist, MS0021570_1 significantly attenuated this CNO-mediated increase in food intake (Supplementary Figure S2E; $\left.t_{(19)}=3.24 ; p<0.001\right)$.

In order to understand the role of BigLEN-GPR171 system in other behaviors, MS0021570_1 was administered to mice followed by psychiatric- and memory-related tests. Systemic
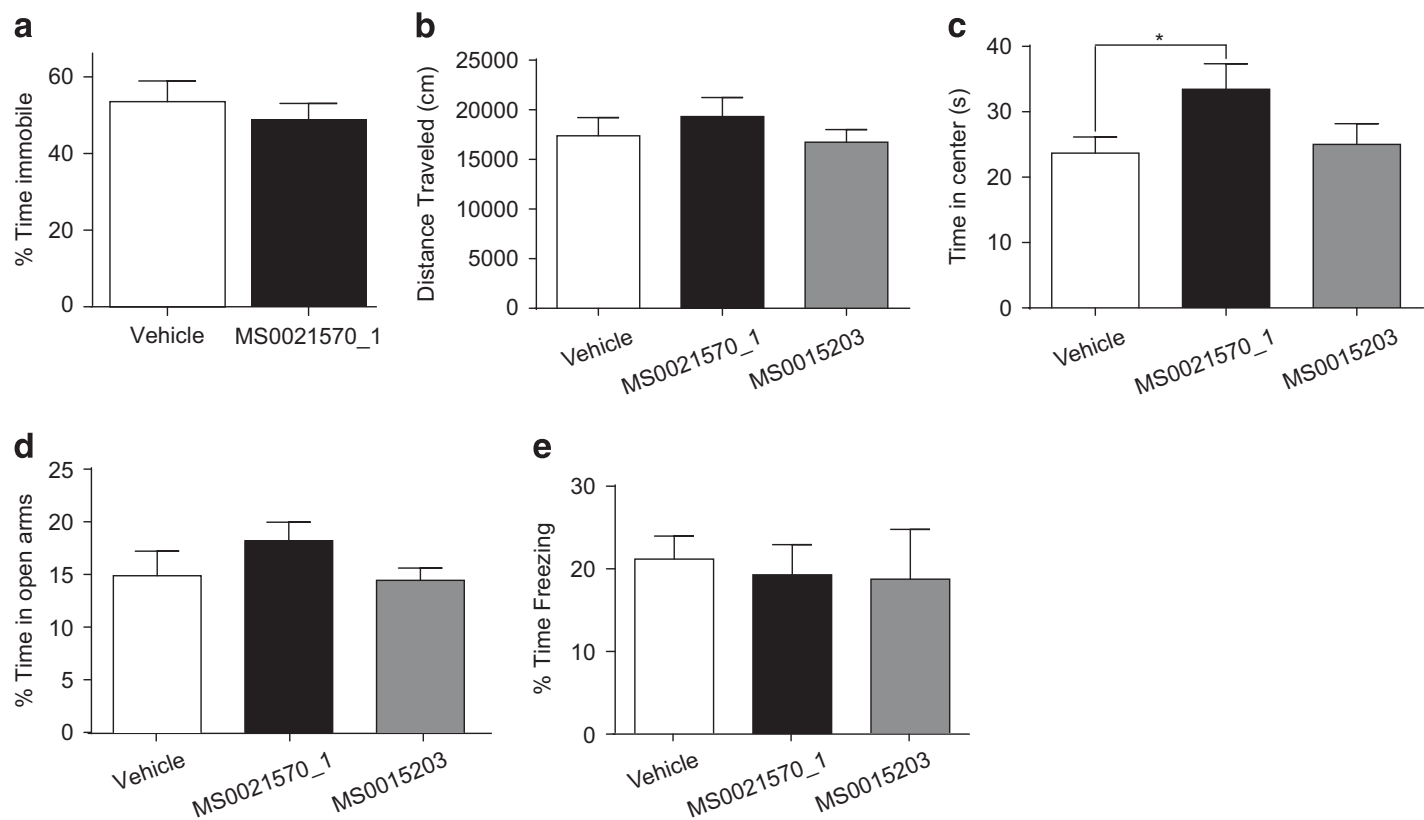

Figure 2 Systemic administration of GPRI7I antagonist decreases anxiety-like behaviors, but not depression and fear. (a) Systemic administration of GPRI7I antagonist, MS0021570_I (5 mg/kg, i.p.; $n=8)$, did not alter depression-like behavior on the forced swim test compared to vehicle-treated mice $(n=7)$. (b) Systemic administration of MSO021570_I $(n=8)$ or GPRI7I agonist, MSO0I5203 (5 mg/kg, i.p.; $n=8)$, did not alter locomotor activity over 30 min test compared to vehicle controls $(n=8)$. (c) MS002I570_I $(n=12)$, but not MSO0I5203 $(n=13)$ increased time in the center on the open field test compared to vehicle $(n=11)$. (d) Systemic administration of MS002I570_I $(n=13)$ or MSO0I5203 $(n=9)$ produce no change in anxiolytic-like behavior on the elevated plus maze compared to vehicle-injected mice $(n=13)$. (e) Systemic injection of MS002I570_l $(n=16)$ or MS00 I5203 ( $n=7)$ when given prior to fear conditioning did not alter freezing behavior $24 \mathrm{~h}$ later compared to vehicle-injected mice $(n=1 \overline{6})$. $* 0.05$. 
injection of the GPR171 antagonist, MS0021570_1 (5 mg/kg, i.p.), had no significant effect on immobility on the forced swim test compared to vehicle (Figure 2a; $t_{(13)}=0.69$, $p=0.50)$. Neither this antagonist, nor the agonist, MS0015203, altered the distance traveled on the open field test (Figure $\left.2 \mathrm{~b}, \mathrm{~F}_{(2,21)}=0.63, p=0.54\right)$. Interestingly, MS0021570_1 caused a significant increase in center time compared to vehicle (Figure 2c; Fisher's LSD; $t_{(33)}=2.07$, $p<0.05)$ suggesting an effect on the anxiety-like behavior; the agonist MS0015203 had no effect (Fisher's LSD; $\left.t_{(33)}=0.29, p=0.77\right)$. In the elevated plus maze, there was no change in the time spent in the open arm following administration of MS0021570_1 or MS0015203 compared to vehicle controls (Figure 2d; $F_{(2,33)}=1.10, p=0.35$ ). Similarly, systemic administration of MS0021570_1 or MS0015203 prior to contextual fear conditioning did not alter freezing $24 \mathrm{~h}$ post shock (Figure 2e; $\mathrm{F}_{(2,36)}=0.11, p=0.89$ ). Together, these results showing that the administration of the agonist had no effect on the majority of the behaviors tested suggest a potentially high basal tone (BigLEN and other ProSAASderived peptides are among the most abundant neuropeptides in the brain). In contrast we find that systemic administration of the GPR171 antagonist reduces AgRP neuron-induced feeding and some anxiety-like behaviors (but has no effect on depression-like behaviors or fear conditioning), suggesting a role for this receptor in modulating anxiety-like behaviors.

A potential site of action for the GPR171 antagonist to regulate anxiety-like behavior is the BLA since it exhibits GPR171 expression (Figure 3a). To test whether blocking GPR171 in the BLA alters behavior, MS0021570_1 was injected bilaterally into BLA; cannula placements are shown in Figure 3b. Intra-BLA administration of MS0021570_1 led to an increase in open arm time on the elevated plus maze (Figure $3 c ; t_{(29)}=2.10 ; p<0.05$ ) although there was no effect on locomotor activity during this test (Supplementary Figure S3A; $t_{24}=1.61, p=0.12$ ). Interestingly, when administered before and immediately following fear conditioning, MS0021570_1 significantly attenuated freezing measured $24 \mathrm{~h}$ later (Figure 3d; $t_{(20)}=2.18 ; p=0<0.05$ ). There was not a significant difference between vehicle and MS0021570_1 when injected outside of BLA on the elevated plus maze (Supplementary Figure S3B; $t_{(13)}=0.15, p=0.88$ ) or contextual fear conditioning (Supplementary Figure S3C; $t_{(9)}=1.11, p=0.30$; placements marked with $\mathrm{X}$ in Figure $3 \mathrm{~b}$ ). Behavioral differences between systemic and intra-BLA administration of MS0021570_1 (ie, no significance observed upon systemic administration) could be due to the pharmacokinetic and pharmacodynamic properties of the drug. Further studies are needed to characterize the GPR171 antagonist. Nevertheless, these results support the notion that antagonism of GPR171 in the BLA leads to a significant decrease in anxiety-like behavior and also in contextual fear conditioning.

\section{Knockdown of GPR171 in the BLA}

If the behavioral effects observed following the administration of MS0021570_1 into the BLA are due to blocking the activity of GPR171, then knocking down these receptors should result in similar effects. To assess this, lentivirus shRNA targeting GPR171 or control lentivirus were

a
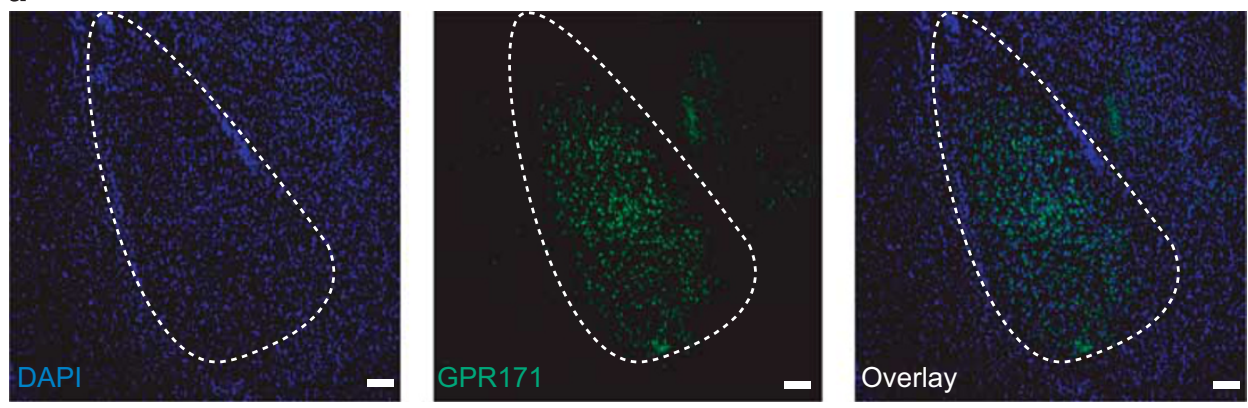

b

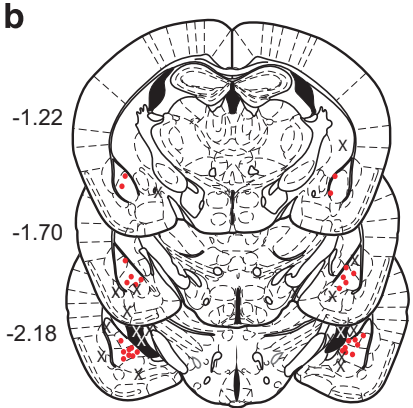

C

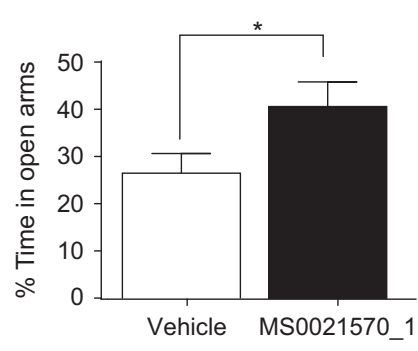

d

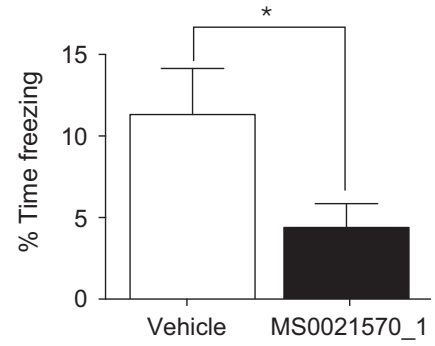

Figure 3 BLA administration of GPR I7I antagonist attenuates anxiety-like behaviors and contextual fear conditioning. (a) GPR I7I (green) is expressed in the basolateral amygdala, outlined area. DAPI is shown in blue. Scale bars $=100 \mu \mathrm{m}$. (b) Distribution of canulla placements in BLA (red dots) and missed placements (Xs). Numbers on left indicate distance from Bregma. (c) Bilateral injection of MS002I570_I (20 nmol/0.5 $\mu$ l; $n=16)$ into the BLA caused a nonsignificant increase in open arm time on elevated plus maze compared to vehicle-injected mice $(n=15)$. (d) Intra-BLA injection of MS002I570_I (20 nmol/ $0.5 \mu l ; n=1 \mathrm{I}$ ) given during acquisition of the fear conditioning caused a decrease in freezing behavior $24 \mathrm{~h}$ after foot shock compared to vehicle-injected mice $(n=\mid I) . * 0<0.05$. BLA, basolateral amygdala. 
a

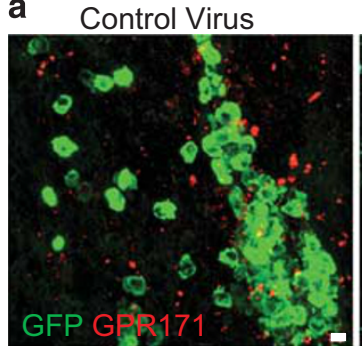

d

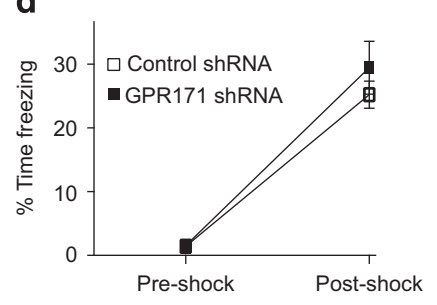

GPR171 shRNA

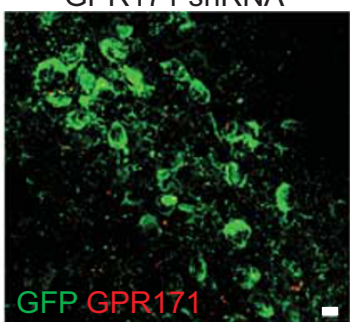

e

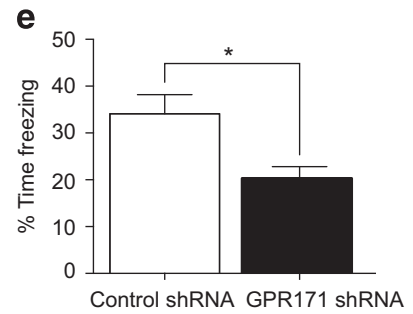

b

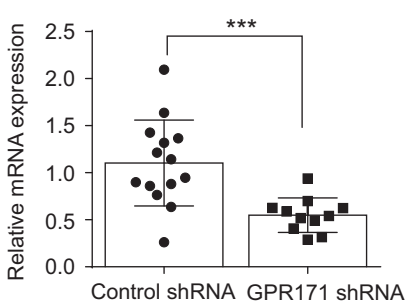

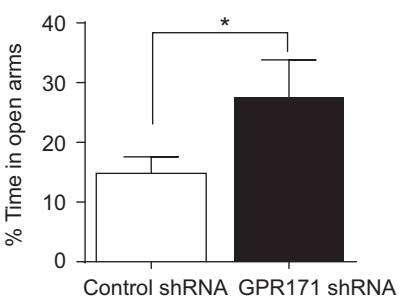

f

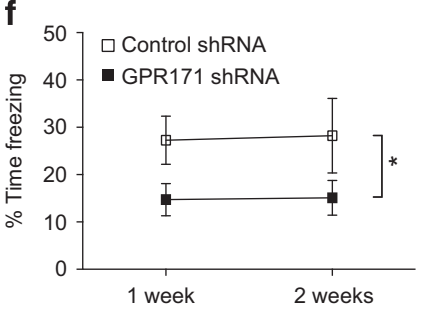

Figure 4 Knockdown of GPRI7I in the basolateral amygdala attenuates anxiety-like behavior and fear conditioning. (a) Mice were injected with control GFP-tagged shRNA (left) or GFP-tagged GPRI7I shRNA lentivirus (right) into the basolateral amygdala. Immunohistochemical staining double labeled with antibodies targeting GPR I 7 I (red) and GFP (green). (b) Quantification of GPR I 7I mRNA using qRT-PCR from mice injected with control or GPR I 7 I shRNA lentivirus in the basolateral amygdala. (c) GPRI7I shRNA $(n=11)$ caused an increase in open arm time on the elevated plus maze compared to mice with control shRNA $(n=15)$. (d) Mice tested on contextual fear conditioning were placed into a novel chamber for 2 min followed by a foot shock. In the 2 min post shock, mice with GPR I7I knockdown in the BLA showed similar freezing as controls. (e) Mice were placed back into the chamber $24 \mathrm{~h}$ later and freezing response was measured for 5 min. Mice with GPRI7I knockdown in BLA ( $n=15)$ showed an attenuation of freezing compared to controls ( $n=19)$. ( $f$ ) Reduced freezing behavior in GPRI7I knockdown mice $(n=8)$ compared to controls $(n=10)$ is seen at I and 2 weeks post shock. Scale bars $=10$ mm ${ }^{*} p<0.05$; **** $p<0.00$ I. BLA, basolateral amygdale; mRNA, messenger RNA; qRT, quantitative real-time; shRNA, short hairpin RNA.

bilaterally infused into the BLA (Figure 4a); this results in $\sim 50 \%$ knockdown of mRNA as detected using qPCR (Figure 4b). When tested for behavior, mice with GPR171 knockdown in BLA spend an increased amount of time in the open arm of the elevated plus maze (Figure 4c; $\left.t_{(24)}=2.05, p<0.05\right)$ with no change in locomotor activity (Supplementary Figure S4A; $t_{(24)}=0.50, p=0.64$ ), center time on the open field test (Supplementary Figure S4B; $t_{(24)}=0.11, p=0.91$ ), and number of marbles buried on the marble burying test (Supplementary Figure S4C; $t_{(15)}=0.35$, $p=0.72$ ). In addition, mice with knockdown of GPR171 in BLA did not show altered depression-like behaviors on forced swim test (Supplementary Figure S4D; $t_{(15)}=0.19$, $p=0.85$ ) or sucrose preference test (Supplementary Figure S4E; $t_{(16)}=0.11, p=0.91$ ). These results further support a role for GPR171 in modulating some anxiety-like behaviors.

When tested in fear conditioning, mice with a knockdown of GPR171 in the BLA freeze immediately following a single foot shock similar to controls (Figure $4 \mathrm{~d} ; \mathrm{F}_{(1,30)}=0.85$, $p=0.36$ ) indicating similar initial fear responses in both groups. However, $24 \mathrm{~h}$ following the shock, mice with BLA GPR171 knockdown show reduced freezing compared to controls (Figure 4e; $t_{(32)}=2.71, p<0.05$ ). This attenuation in contextual fear conditioning was found to be maintained for up to 2 weeks (Figure $4 \mathrm{f} ; \mathrm{F}_{(1,32)}=5.04, p<0.05$ ). Together, these results suggest that GPR171 in BLA regulates some anxiety-like behaviors and contextual fear conditioning.

\section{Characterization of GPR171 in the BLA}

Given that GPR171 signals through $\mathrm{G}_{\mathrm{i} / \mathrm{o}}$ in the hypothalamus (Gomes et al, 2013), we hypothesized that GPR171 activation may lead to membrane hyperpolarization within the BLA. We tested this by continuously recording the membrane potential of BLA pyramidal neurons while both applying either BigLEN or a small molecule GPR171 agonist (MS0015203). We observed a significant hyperpolarization in response to both BigLEN (Figure $5 \mathrm{a} ;-73.6 \pm 1.5 \mathrm{mV}$, $\mathrm{F}_{(2,10)}=10.84, \quad p<0.004$ ) and MS0015203 (Figure 5b; $\left.-73.5 \pm 0.6 \mathrm{mV}, \quad \mathrm{F}_{(2,14)}=21.27, p<0.0001\right)$ compared to baseline (BigLEN, $\quad-69.8 \pm 0.5 \mathrm{mV}$; MS0015203, $-70.2 \pm 0.2 \mathrm{mV})$. Upon addition of the GPR171 antagonist (MS0021570_1) in the presence of either BigLEN or MS0015203, we observed a return to baseline-like potentials (BigLEN+MS0021570_1, $\quad-69.5 \pm 0.4 \mathrm{mV} ; \quad$ MS0015203 +MS0021570_1, $-69.4 \pm 0.4 \mathrm{mV})$. We also found that $1 \mu \mathrm{M}$ BigLEN and $1 \mu \mathrm{M}$ MS0015203 cause inhibition of cAMP levels, which is blocked by application of pertussis toxin (Figure 5c). Immunohistochemical analysis showed that GPR171 is found on vGlut2- and GAD67-positive neurons within the BLA (Supplementary Figure S5). These results indicate that GPR171 is coupled to Goi/o, which leads to membrane potential hyperpolarization following GPR171 agonist application on pyramidal neurons, however GPR171 is found on both excitatory and inhibitory neurons.

\section{DISCUSSION}

In this study, we show a role for the BigLEN-GPR171 system in fear and anxiety-like behaviors using a small molecule antagonist, MS0021570_1 and viral knockdown of GPR171. To identify the antagonist of GPR171, we used the crystal structure of a phylogenetically related receptor (P2Y12), to generate a homology model of GPR171 and virtually 
a

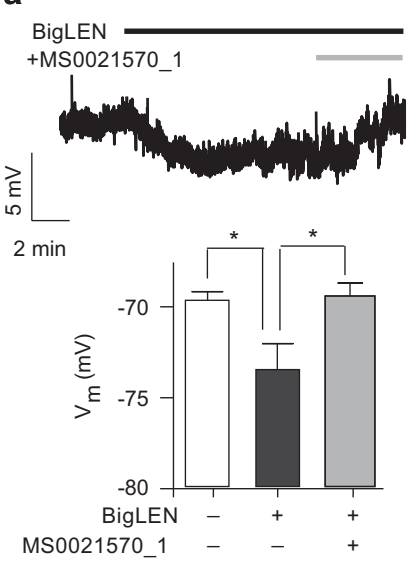

\section{b}

MS0015203

+MS0021570_1

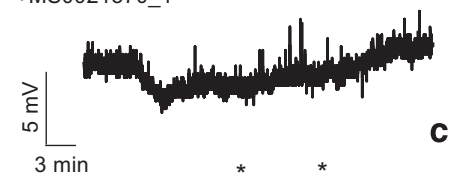

$3 \mathrm{~min}$

C

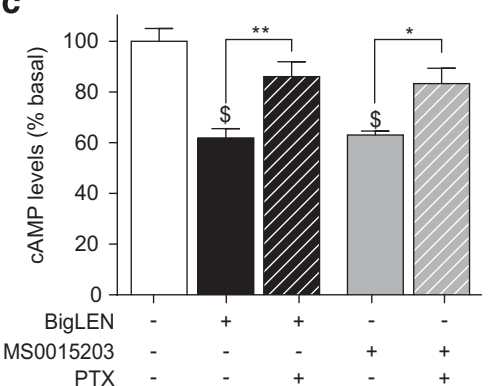

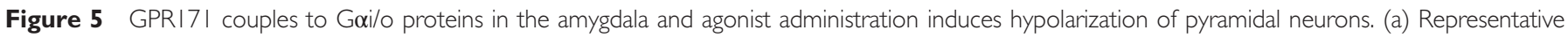

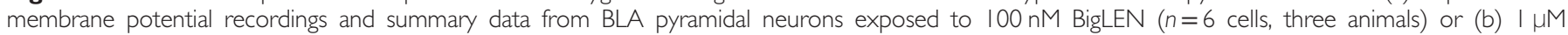

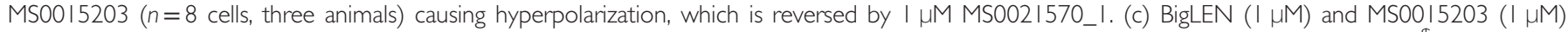

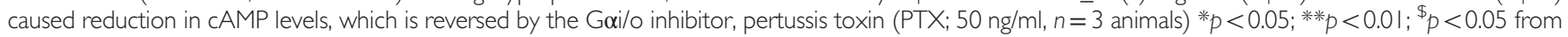
basal (white bar). BLA, basolateral amygdala.

screened for putative receptor ligands. This approach has been successfully used to identify novel ligands with unique structures that bind to a given receptor, such as antagonists to melanin-concentrating hormone receptor 1 (Cavasotto et al, 2008) and allosteric modulators of mGluR5 (Anighoro et al, 2015). Thus, the crystal structure of a receptor or the homology model of a receptor based on the crystal structure of a closely related receptor can be used to identify ligands by virtual screening (Stockert and Devi, 2015). We previously used this strategy to identify MS0015203 as an agonist targeting GPR171 (Wardman et al, 2016); in this study a similar strategy was used to identify MS0021570_1 as an antagonist. The advantage of such an approach is the unbiased nature of ligand identification as well as the possibility of identifying molecules with novel scaffolds that could be used for modifying the hits to lead to identification of compounds that serve as invaluable tools for the pharmacological characterization of GPCRs.

In order to characterize the specificity of MS0021570_1, we subjected it to a screen consisting of $\sim 80$ different membrane proteins and among them MS0021570_1 was found to bind to only one other GPCR, MT1AR receptor. However, further characterization revealed that only at high concentration MS0021570_1 could function as an agonist of this receptor. Thus, it is possible that a portion of the behavioral effects observed with this ligand are partially due to its agonistic effects at MT1AR. However, this is unlikely since localization studies (autoradiography with ${ }^{125} \mathrm{I}$ radiolabeled melatonin) have revealed that this receptor is not expressed in the amygdala (Morgan et al, 1994) - the focus of the current study. Furthermore, our electrophysiological data with pyramidal BLA neurons show that MS0021570_1 antagonizes the effects of the small molecule agonist MS0015203 that does not exhibit binding to melatonin receptors (Wardman et al, 2016). Finally, the observation that knockdown of GPR171 in the BLA produces behavioral effects similar to those seen upon administration of MS0021570_1 supports that this compound mediates its effects via GPR171.
The identification of MS0021570_1 as a selective antagonist was useful in exploring the role of GPR171 in anxietylike behaviors and in fear memories. It is well established that the amygdala is important for the acquisition, consolidation, and retrieval of fear memories and the maintenance of maladaptive behaviors such as anxiety (Maren et al, 2013). Specifically, the BLA integrates sensory information and plays a central role in responding to fearful stimuli and the maintenance of contextual fear memories (Kochli et al, 2015). The activity of the BLA is tightly regulated by a combination of excitatory glutamatergic and inhibitory GABAergic signals. The pyramidal neurons in the BLA are glutamatergic (Carlsen, 1988; Smith and Pare, 1994), while local circuit interneurons are GABAergic (McDonald and Augustine, 1993). Studies show that acute and chronic stress cause an increase in excitability of BLA pyramidal neurons (Hetzel and Rosenkranz, 2014; Rau et al, 2015), and decreasing BLA excitability causes a reduction in anxiety-like behaviors (Silberman et al, 2010). This is the first study to show that activation of GPR171 in the BLA leads to hyperpolarization of pyramidal neurons. It is also possible that GPR171 could modulate anxiety and fear via different subregions of the amygdala. Further studies are needed to determine how GPR171 alters excitability of these neurons and whether it is a presynaptic or postsynaptic mechanism. Our behavioral studies show that both antagonist administration and knockdown of GPR171 in BLA leads to attenuation of anxiety-like behavior and fear conditioning. Given that we found that GPR171 is found on GAD67positive neurons in addition to the vGlut-positive pyramidal neurons, it is likely that GPR171 antagonist microinjection and knockdown in the BLA hit both populations of neurons. It will be important for future studies to knockdown GPR171 in a cell type-specific manner to elucidate the role of GPR171 in these behaviors.

The BLA is part of a larger circuitry that modulates fear and anxiety-like behaviors, which includes reciprocal projections from the medial prefrontal cortex (mPFC) and ventral 
hippocampus (vHPC) (Felix-Ortiz et al, 2013; Maren et al, 2013) as well as projections to the central amygdala (Gafford and Ressler, 2016). Since antagonizing or knocking down GPR171 in the BLA has effects on anxiety-like behaviors and fear conditioning, it would be important for future studies to investigate the involvement of BigLEN and GPR171 in mPFC, vHPC, and central amygdala in fear and anxiety-like behaviors. The origin of fibers that contain BigLEN that act on pyramidal cells within the BLA is unknown. Given colocalization of BigLEN with NPY in the hypothalamus (Wardman et al, 2011), it is possible that they are colocalizaed in other brain regions as well. Recent studies have shown that NPY projections into the BLA originate from multiple regions, which seem to regulate fear conditioning (Leitermann et al, 2016). It is possible that NPY and BigLEN within the BLA are working in opposition to maintain homeostasis, for example, BigLEN release increases anxiety and NPY decreases anxiety.

Neuropeptides and their receptors play a role in modulating fear and anxiety-like behaviors in the BLA including NPY, PACAP, corticotrophin releasing factor (Hashimoto et al, 2001; Otto et al, 2001; Tasan et al, 2010; Cipriano et al, 2016). Our data show that within the BLA the BigLENGPR171 system also plays a role. Support for this also comes from peptidomic analysis that detect BigLEN in several key brain regions involved in fear and anxiety-like behaviors, including amygdala, hippocampus, and prefrontal cortex (Zhang et al, 2008). A previous study in ProSAAS (precursor of BigLEN) knockout mice show a decrease in locomotor activity, which was attributed to altered anxiety (Morgan et al, 2010). However, we do not see a change in activity following our antagonist treatment or knockdown of GPR171. This difference can be explained by the multiple other neuropeptides that are missing in the ProSASS knockout mice (ie, PEN, SAAS) in addition to increases in compensatory systems. Further studies mapping the localization and function of BigLEN and GPR171 throughout the brain would help in understanding how this system modulates fear and anxiety-like behaviors.

Taken together these results suggest that the BigLENGPR171 system facilitates anxiety and fear conditioning. Systemic, intra-BLA administration of the GPR171 antagonist or knockdown of BLA GPR171 in the BLA causes a reduction in some anxiety-like behaviors and in fear conditioning. Anxiety and fear conditioning are complicated behaviors involving multiple brain regions, but disrupting GPR171 function within the BLA is sufficient to block the output of these behaviors. In conclusion, the BigLENGPR171 system is a new system that contributes to a variety of physiological functions including feeding, fear, and anxiety-like behaviors. This exciting new research expands our knowledge of neuropeptides in these behaviors.

\section{FUNDING AND DISCLOSURE}

This work was supported by National Institute Health Awards DA008863 and NS026880 to LAD, MH105414 to RLC, MH096678 to KAC, and T32 DA007135 and a NARSAD Young Investigator Grant from Brain and Behavior Research Foundation to ENB. The authors declare no conflict of interest.

\section{ACKNOWLEDGMENTS}

We thank Dr Marta Filizola and Dr Abhijeet Kapoor for generation of the homology model of GPR171 and members of the Devi lab for active scientific discussions.

\section{REFERENCES}

Anighoro A, Graziani D, Bettinelli I, Cilia A, De Toma C, Longhi M et al (2015). Insights into the interaction of negative allosteric modulators with the metabotropic glutamate receptor 5: discovery and computational modeling of a new series of ligands with nanomolar affinity. Bioorg Med Chem 23: 3040-3058.

Carlsen J (1988). Immunocytochemical localization of glutamate decarboxylase in the rat basolateral amygdaloid nucleus, with special reference to GABAergic innervation of amygdalostriatal projection neurons. J Comp Neurol 273: 513-526.

Cavasotto CN, Orry AJ, Murgolo NJ, Czarniecki MF, Kocsi SA, Hawes BE et al (2008). Discovery of novel chemotypes to a G-protein-coupled receptor through ligand-steered homology modeling and structure-based virtual screening. J Med Chem 51: 581-588.

Christiansen SH, Olesen MV, Gotzsche CR, Woldbye DP (2014). Anxiolytic-like effects after vector-mediated overexpression of neuropeptide $\mathrm{Y}$ in the amygdala and hippocampus of mice. Neuropeptides 48: 335-344.

Cipriano AC, Gomes KS, Nunes-de-Souza RL (2016). CRF receptor type 1 (but not type 2 ) located within the amygdala plays a role in the modulation of anxiety in mice exposed to the elevated plus maze. Horm Behav 81: 59-67.

Desai SJ, Upadhya MA, Subhedar NK, Kokare DM (2013). NPY mediates reward activity of morphine, via NPY Y1 receptors, in the nucleus accumbens shell. Behav Brain Res 247: 79-91.

Felix-Ortiz AC, Beyeler A, Seo C, Leppla CA, Wildes CP, Tye KM (2013). BLA to vHPC inputs modulate anxiety-related behaviors. Neuron 79: 658-664.

Fricker LD (2010). Analysis of mouse brain peptides using mass spectrometry-based peptidomics: implications for novel functions ranging from non-classical neuropeptides to microproteins. Mol Biosyst 6: 1355-1365.

Gafford GM, Ressler KJ (2016). Mouse models of fear-related disorders: cell-type-specific manipulations in amygdala. Neuroscience 321: 108-120.

Gloriam DE, Fredriksson R, Schioth HB (2007). The G proteincoupled receptor subset of the rat genome. BMC Genomics 8: 338.

Gomes I, Aryal DK, Wardman JH, Gupta A, Gagnidze K, Rodriguiz RM et al (2013). GPR171 is a hypothalamic G protein-coupled receptor for BigLEN, a neuropeptide involved in feeding. Proc Natl Acad Sci USA 110: 16211-16216.

Gomes I, Filipovska J, Devi LA (2003). Opioid receptor oligomerization. Detection and functional characterization of interacting receptors. Methods Mol Med 84: 157-183.

Gomes I, Grushko JS, Golebiewska U, Hoogendoorn S, Gupta A, Heimann AS et al (2009). Novel endogenous peptide agonists of cannabinoid receptors. FASEB J 23: 3020-3029.

Gupta A, Mulder J, Gomes I, Rozenfeld R, Bushlin I, Ong E et al (2010). Increased abundance of opioid receptor heteromers after chronic morphine administration. Sci Signal 3: ra54.

Hashimoto H, Shintani N, Tanaka K, Mori W, Hirose M, Matsuda T et al (2001). Altered psychomotor behaviors in mice lacking pituitary adenylate cyclase-activating polypeptide (PACAP). Proc Natl Acad Sci USA 98: 13355-13360.

Hetzel A, Rosenkranz JA (2014). Distinct effects of repeated restraint stress on basolateral amygdala neuronal membrane properties in resilient adolescent and adult rats. Neuropsychopharmacology 39: 2114-2130. 
Hodes GE, Pfau ML, Purushothaman I, Ahn HF, Golden SA, Christoffel DJ et al (2015). Sex differences in nucleus accumbens transcriptome profiles associated with susceptibility versus resilience to subchronic variable stress. $J$ Neurosci 35: $16362-16376$.

Kakarala KK, Jamil K (2014). Sequence-structure based phylogeny of GPCR class A rhodopsin receptors. Mol Phylogenet Evol 74: 66-96.

Kochli DE, Thompson EC, Fricke EA, Postle AF, Quinn JJ (2015). The amygdala is critical for trace, delay, and contextual fear conditioning. Learn Mem 22: 92-100.

Krashes MJ, Koda S, Ye C, Rogan SC, Adams AC, Cusher DS et al (2011). Rapid, reversible activation of AgRP neurons drives feeding behavior in mice. J Clin Invest 121: 1424-1428.

Lein ES, Hawrylycz MJ, Ao N, Ayres M, Bensinger A, Bernard A et al (2007). Genome-wide atlas of gene expression in the adult mouse brain. Nature 445: 168-176.

Leitermann RJ, Rostkowski AB, Urban JH (2016). Neuropeptide Y input to the rat basolateral amygdala complex and modulation by conditioned fear. J Comp Neurol 524: 2418-2439.

Maren S, Phan KL, Liberzon I (2013). The contextual brain: implications for fear conditioning, extinction and psychopathology. Nat Rev Neurosci 14: 417-428.

McDonald AJ, Augustine JR (1993). Localization of GABA-like immunoreactivity in the monkey amygdala. Neuroscience 52: 281-294.

Morgan DJ, Wei S, Gomes I, Czyzyk T, Mzhavia N, Pan H et al (2010). The propeptide precursor proSAAS is involved in fetal neuropeptide processing and body weight regulation. I Neurochem 113: 1275-1284.

Morgan PJ, Barrett P, Howell HE, Helliwell R (1994). Melatonin receptors: localization, molecular pharmacology and physiological significance. Neurochem Int 24: 101-146.

Otto C, Martin M, Wolfer DP, Lipp HP, Maldonado R, Schutz G (2001). Altered emotional behavior in PACAP-type-I-receptordeficient mice. Brain Res Mol Brain Res 92: 78-84.

Paxinos G, Franklin KBJ (2001). The Mouse Brain in Stereotaxic Coordinates. Academic Press: San Diego, CA.

Piccoli G, Onofri F, Cirnaru MD, Kaiser CJ, Jagtap P, Kastenmuller A et al (2014). Leucine-rich repeat kinase 2 binds to neuronal vesicles through protein interactions mediated by its C-terminal WD40 domain. Mol Cell Biol 34: 2147-2161.

Rau AR, Chappell AM, Butler TR, Ariwodola OJ, Weiner JL (2015). Increased basolateral amygdala pyramidal cell excitability may contribute to the anxiogenic phenotype induced by chronic early-life stress. J Neurosci 35: 9730-9740.

Rozenfeld R, Gupta A, Gagnidze K, Lim MP, Gomes I, Lee-Ramos D et al (2011). AT1R-CB(1)R heteromerization reveals a new mechanism for the pathogenic properties of angiotensin II. EMBO J 30: 2350-2363.

Sajdyk TJ, Johnson PL, Leitermann RJ, Fitz SD, Dietrich A, Morin $\mathrm{M}$ et al (2008). Neuropeptide $\mathrm{Y}$ in the amygdala induces long-term resilience to stress-induced reductions in social responses but not hypothalamic-adrenal-pituitary axis activity or hyperthermia. J Neurosci 28: 893-903.

Silberberg D, Anand NP, Michels K, Kalaria RN (2015). Brain and other nervous system disorders across the lifespan - global challenges and opportunities. Nature 527: S151-S154.

Silberman Y, Ariwodola OJ, Chappell AM, Yorgason JT, Weiner JL (2010). Lateral paracapsular GABAergic synapses in the basolateral amygdala contribute to the anxiolytic effects of beta 3 adrenoceptor activation. Neuropsychopharmacology 35: 1886-1896.

Smith Y, Pare D (1994). Intra-amygdaloid projections of the lateral nucleus in the cat: PHA-L anterograde labeling combined with postembedding GABA and glutamate immunocytochemistry. J Comp Neurol 342: 232-248.

Stamatakis AM, Sparta DR, Jennings JH, McElligott ZA, Decot H, Stuber GD (2014). Amygdala and bed nucleus of the stria terminalis circuitry: implications for addiction-related behaviors. Neuropharmacology 76(Pt B): 320-328.

Stockert JA, Devi LA (2015). Advancements in therapeutically targeting orphan GPCRs. Front Pharmacol 6: 100.

Tasan RO, Nguyen NK, Weger S, Sartori SB, Singewald N, Heilbronn $\mathrm{R}$ et al (2010). The central and basolateral amygdala are critical sites of neuropeptide $\mathrm{Y} / \mathrm{Y} 2$ receptormediated regulation of anxiety and depression. J Neurosci 30: 6282-6290.

Wardman JH, Berezniuk I, Di S, Tasker JG, Fricker LD (2011). ProSAAS-derived peptides are colocalized with neuropeptide Y and function as neuropeptides in the regulation of food intake. PLoS ONE 6: e28152.

Wardman JH, Gomes I, Bobeck EN, Stockert JA, Kapoor A, Bisignano $P$ et al (2016). Identification of a small-molecule ligand that activates the neuropeptide receptor GPR171 and increases food intake. Sci Signal 9: ra55.

Zhang X, Che FY, Berezniuk I, Sonmez K, Toll L, Fricker LD (2008). Peptidomics of Cpe(fat/fat) mouse brain regions: implications for neuropeptide processing. J Neurochem 107: 1596-1613.

Supplementary Information accompanies the paper on the Neuropsychopharmacology website (http://www.nature.com/npp) 